\title{
Edaravone Diminishes Free Radicals from Circulating Neu- trophils in Patients with Ischemic Brain Attack
}

\author{
Hitoshi Aizawa ${ }^{1}$, Yoshihiro Makita ${ }^{1}$, Kazuhiro Sumitomo ${ }^{1}$, Yoko Aburakawa ${ }^{1}$, \\ Takayuki Katayama ${ }^{1}$, Setsu Nakatani-Enomoto ${ }^{1}$, Yasuhiro Suzuki ${ }^{1}$, Kazuhiko Fujiwara ${ }^{2}$, \\ Hiroyuki Enomoto $^{2}$, Kenji Kuroda ${ }^{2}$, Takashi Kimura ${ }^{2}$, Osamu Yahara $^{2}$, Satoshi Koyama ${ }^{3}$, \\ Junichi Maruyama $^{3}$, Masao Nakamura ${ }^{4}$, Naoyuki Hasebe ${ }^{1}$ and Kenjiro Kikuchi ${ }^{1}$
}

\begin{abstract}
Objective Treatment with a free radical scavenger could be a new option for ischemic brain attack, however, little is known about the alteration of oxidative stress markers induced by edaravone, a novel free radical scavenger, in human ischemic brain attack.

Methods We investigated the effects of edaravone on the oxidative stress markers in patients with ischemic brain attack. Twenty-one patients with ischemic brain attack and 19 controls were enrolled in this study. Blood samples were obtained just before and soon after the first administration of edaravone (30 mg) or ozagrel (40 mg). Intracellular reactive oxygen species of neutrophils were measured using 6-carboxy-2', 7'dichlorodihydrofluorescin diacetate and a fluorescence-activated cell sorter. Superoxide from neutrophils, induced by phorbol myristate acetate (PMA), was determined by luminol-amplified chemiluminescence assay. Results Treatment with $30 \mathrm{mg}$ of edaravone significantly decreased the intracellular reactive oxygen species (ROS) of neutrophils (Wilcoxon test, $\mathrm{p}=0.0001$ ) and PMA-induced superoxide produced by neutrophils (Wilcoxon test, $\mathrm{p}=0.001$ ). Ozagrel did not alter the intracellular ROS or superoxide production of neutrophils.

Conclusion Reduction of intracellular ROS and suppression of superoxide production in neutrophils provide a potential explanation for the clinical efficacy of edaravone in patients with ischemic brain attack.
\end{abstract}

Key words: edaravone, free radical scavenger, reactive oxygen species, neutrophil, ischemic brain attack

(DOI: 10.2169/internalmedicine.45.1491)

\section{Introduction}

Accumulating evidence implicates free radicals in the damage of ischemic brain attack, and free radical scavengers have been proposed as a new clinical strategy. A novel free radical scavenger, edaravone (MCI-186, 3-methyl-1-phenyl2-pyrazolin-5-one), has been shown to prevent vascular endothelial injury in vitro (1) and cerebral damage following middle cerebral artery occlusion (2), delayed neuronal death in transient cerebral ischemia (3) and ischemic brain edema in rats $(4,5)$. Edaravone has been found to inhibit activation of the lipoxygenase pathway in the arachidonic acid cascade
(6) and peroxidation of the phosphatidylcholine liposomal membrane (7), as well as to scavenge hydroxyradicals.

The clinical efficacy of edaravone on ischemic brain attack has been demonstrated by significant improvement in the functional outcome in a randomized, placebo-controlled, double-blind study (8). However, to date no data is available on the alteration of oxidative stress markers due to edaravone in human ischemic brain attack. We investigated the effects of edaravone on oxidative stress markers of circulating neutrophils in patients with ischemic brain attack.

\footnotetext{
${ }^{1}$ The First Department of Medicine, Asahikawa Medical College, ${ }^{2}$ Department of Neurology, National Douhoku Hospital, ${ }^{3}$ Asahikawa Rehabilitation Hospital and ${ }^{4}$ Department of Chemistry, Asahikawa Medical College, Asahikawa

Received for publication July 11, 2005; Accepted for publication November 2, 2005

Reprint requests should be addressed to Hitoshi Aizawa, The First Department of Medicine, Asahikawa Medical College, 2-1-1-1 MidorigaokaHigashi, Asahikawa, Hokkaido 078-8510
} 
A

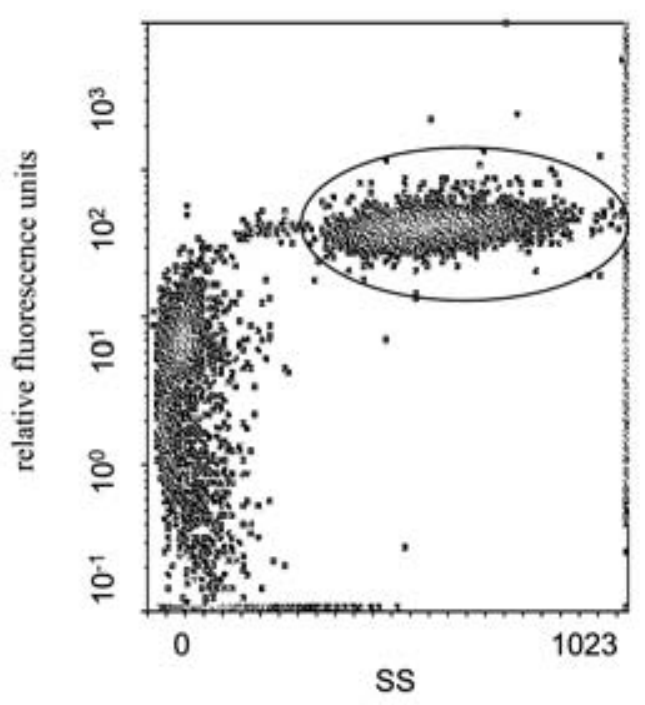

B

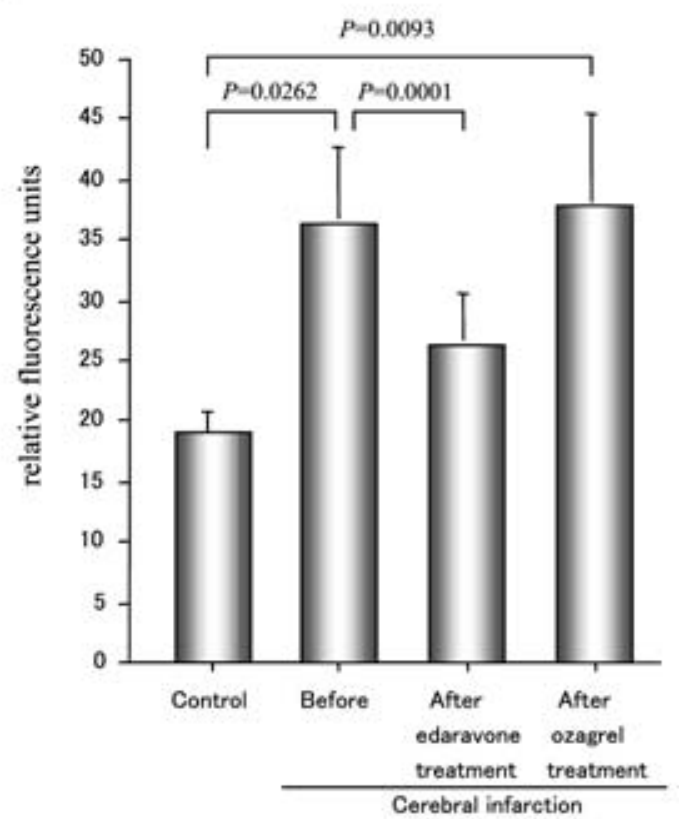

Figure 1. Intracellular reactive oxygen species of circulating neutrophils in patients with ischemic brain attack and controls. A: Typical flow cytogram of neutrophils (surrounded by an oval). Intracellular reactive oxygen species are presented as relative fluorescence units, i.e., the median channel value of the fluorescing cell population. SS = side scatter dot plot. B: Relative fluorescence units of neutrophils before and after treatment with $30 \mathrm{mg}$ of edaravone or $\mathbf{4 0} \mathbf{~ m g}$ of ozagrel.

\section{Patients and Methods}

Twenty-one patients (14 males and 7 females, mean \pm SD; $71 \pm 2$ years old, range 52-88) with ischemic brain attack and 19 untreated healthy controls (11 males and 7 females, mean $\pm \mathrm{SD}$; 51 \pm 4 years old, range 23-79) were enrolled in this study under informed consent. Edaravone treatment was started for the patients within 24 hours after the onset of ischemic brain attack. Patients were administered $30 \mathrm{mg}$ of edaravone i.v. over 1 hour twice a day for 3-10 days. Ten patients were treated with $40 \mathrm{mg}$ of ozagrel, a thromboxane A2 synthase inhibitor, over 1 hour before the treatment with edaravone. Blood samples were obtained just before and soon after the first administration of edaravone or ozagrel. Glycerol was used for the patients after the first treatment with edaravone.

To measure intracellular reactive oxygen species of neutrophils, venous peripheral blood was incubated in $\mathrm{NH}_{4} \mathrm{Cl}$ $(8.26 \mathrm{~g} / \mathrm{L}) \quad \mathrm{KHCO}_{3}(1.0 \mathrm{~g} / \mathrm{L})$ and tetrasodium ethylenediaminetetraacetic acid $(0.037 \mathrm{~g} / \mathrm{L})$ in Chelex 100-treated water $(\mathrm{pH} 7.3)$ for $10 \mathrm{~min}$ to lyse erythrocytes, washed with ice-cold 2-[4-(2-hydroxyethyl)-1-piperazinyl] ethanesulfonic acid (HEPES) buffer and then centrifuged at $1,000 \mathrm{~g}$ at $4^{\circ} \mathrm{C}$ for $5 \mathrm{~min}$. The supernatant was discarded. Neutrophils were resuspended in $1 \mathrm{ml}$ HEPES buffer, and 6-carboxy-2', 7'dichlorodihydrofluorescin diacetate (CDCFH-DA;Molecular Probes, Netherlands) (10 $\mu \mathrm{M}$ final concentration) was added to each sample tube; and the tubes were incubated at $37^{\circ} \mathrm{C}$ for $30 \mathrm{~min}$ before data acquisition by flow cytometry. A fluorescence-activated cell sorter (EPICS XL/XL-MCL System II v3.0, Becton Dickinson) and WinMIDI 28 software were used for acquisition and analysis of data. The stability of instrumental settings including spectral compensations was checked regularly with fluorescent beads (Calibrite, Becton Dickinson). For each $1 \mathrm{ml}$ aliquot of cell suspension, an appropriate electronic gate was generated, and 5,000 events were collected in live mode. Neutrophils were delineated in a forward/side scatter dot plot, and appropriate fluorescence histograms were developed. Fluorescence intensities are reported in relative fluorescence units, i.e., the median channel value of the fluorescing cell population (Fig. 1A).

To measure superoxide produced by neutrophils, neutrophils were resuspended in $1 \mathrm{ml}$ of HEPES buffer by the above method and adjusted to the density of $2 \times 10^{5} / \mathrm{ml}$. Superoxide production by phorbol myristate acetate (PMA) was determined by luminol-amplified chemiluminescence assay using a luminometer (BLR-301, ALOKA, Japan). Neutrophils were stimulated with PMA $(0.1 \mathrm{nmol} / \mathrm{L})$, and the resulting light output was continuously recorded on a chart recorder. All results are expressed as kilo-counts per minute (kcpm) using the chemiluminescence emission peak (Fig. 2A).

\section{Results}

The controls showed no significant relationship between 
A

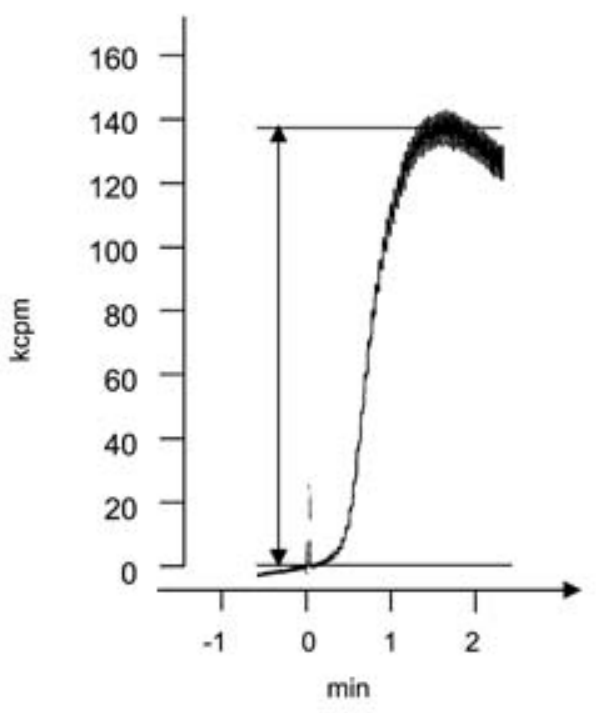

B

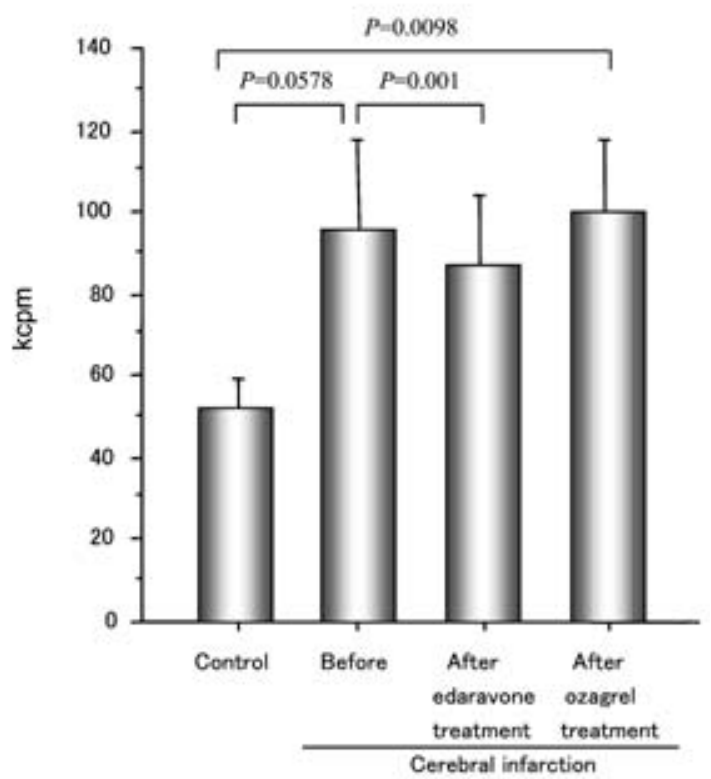

Figure 2. Superoxide production of circulating neutrophils in patients with ischemic brain attack and controls. A: Typical light output continuously recorded on a chart recorder, reflecting amount of superoxide produced by neutrophils stimulated by phorbol myristate acetate (PMA). B: Amount of superoxide produced by neutrophils stimulated by PMA before and after treatment with edaravone or ozagrel. All results are expressed as kilo-counts per minutes (kcpm) using the chemiluminescence emission peak.

age and relative fluorescence units of circulating neutrophils treated with CDCFH (simple regression analysis, $\mathrm{p}=0.1832$ ) or between age and superoxide production by neutrophils ( simple regression analysis, $\mathrm{p}=0.2406$ ). The relative fluorescence units of circulating neutrophils in $\mathrm{CDCFH}$ in patients with ischemic brain attack before treatment with edaravone or ozagrel $(36.8 \pm 5.9$, mean $\pm \mathrm{SE})$ was higher than that of controls $(19.1 \pm 1.8)$ (Mann-Whitney U test, $\mathrm{p}=0.0262)$. The relative fluorescence units in $\mathrm{CDCFH}$ decreased after treatment with edaravone $(26.8 \pm 3.8)$ (Wilcoxon test, $\mathrm{p}=0.0001$ ), indicating that edaravone lowered the intracellular ROS of circulating neutrophils (Fig. 1B). In contrast, the relative fluorescence units in CDCFH showed no significant alteration after the treatment with ozagrel $(38.3 \pm 7.2)$.

Amount of superoxide produced by neutrophils in patients with ischemic brain attack tended to be higher than those of controls (Mann-Whitney U test, $\mathrm{p}=0.0578$ ). The superoxide production by neutrophils decreased after treatment with edaravone in patients with ischemic brain attack (Wilcoxon test, $\mathrm{p}=0.001$; Fig. 2B). The amount of superoxide from neutrophils showed no significant alteration after the treatment of ozagrel. In one patient, we happened to obtain a blood sample one day before ischemic brain attack. The preliminary data showed that the superoxide produced by neutrophils after ischemic brain attack was increased to $135 \%$ of that before ischemic brain attack, indicating an increase in inducible superoxide from neutrophils after ischemic brain attack. No adverse events were observed in patients treated with $30 \mathrm{mg}$ of edaravone.

Discussion

Brain damage in ischemic brain attack is closely related to inflammatory responses, especially infiltration of circulating neutrophils into ischemic tissue (9). Many studies show that inhibition of neutrophil accumulation greatly reduces infarct size $(10,11)$. Neutrophil accumulation in vessels requires interaction between several adhesion molecules, such as intracellular adhesion molecule (ICAM)-1. Interleukin (IL)-1 $\beta$ and tumor necrosis factor- $\alpha$ which upregulate ICAM (12) are increased in ischemic brain tissue. Ischemia induces the formation of IL-1, the subsequent expression of ICAM1 , and increased adherence of neutrophils. In ischemic brain tissue, neutrophil, cytokines, and adhesion molecules are closely related to each other.

Neutrophils are a potential source of ROS when activated during inflammatory responses. Free radicals from the neutrophils are produced via activation of NADPH oxidase in the cytoplasmic membrane. An ischemia and reperfusion animal model demonstrated that the major site of superoxide production is the activated leukocytes in the circulating blood (13). Superoxide and $\mathrm{H}_{2} \mathrm{O}_{2}$ have since been shown to directly trigger leukocyte activation and adhesion to vascular endothelium. Neutrophils might induce damage by causing local vascular occlusion, or they might initiate toxic reactions, including free radical production by NADPH oxidase, production of hypochlorous acid, or protease activation (14). Transgenic mice lacking NADPH oxidase showed reduction 
of infarct size by $50 \%$, however, selective elimination of either leukocyte or parenchymal NADPH oxidase did not reduce damage, suggesting both NADPH oxidases need to be eliminated to protect the brain from ischemia (15). A large part of free radical production is dependent on neutrophils, but it could be initiated by NADPH oxidation in parenchymal tissue or in the neutrophils (14). These findings suggest that inhibition of circulating neutrophils in ischemic brain attack could be effective to prevent ischemic brain injury.

We demonstrated here that edaravone diminished free radicals in circulating neutrophils in patients with ischemic brain attack, which could at least partially explain the clinical efficacy of edaravone on ischemic brain attack (8). We speculate that edaravone eventually protects the ischemic brain by scavenging free radicals from circulating neutrophils as well as from brain tissue.

Acknowledgements: We wish to thank Drs. S. Tsuji and H. Sakuragi (Asahikawa Rehabilitation Hospital) for their cooperation and Kaori Kanno and Mika Yashima (First Department of Medicine, Asahikawa Medical College) for their excellent technical assistance.

\section{References}

1. Watanabe T, Morita I, Nishi H, Murota S. Preventive effect of MCI-186 on 15-HEPETE induced vascular endothelial cell injury in vitro. Prostaglandins Leukot Essent Fatty Acids 33: 81-87, 1988.

2. Kawai H, Nakai H, Suga M, Yuki S, Watanabe T, Saito KI. Effects of a novel free radical scavenger, MCI-186, on ischemic brain damage in the rat distal middle cerebral artery occlusion model. J Pharmacol Exp Ther 281: 921-927, 1997.

3. Yamamoto T, Yuki S, Watanabe T, Mitsuka M, Saito KI, Kogure K. Delayed neuronal death prevented by inhibition of increased hydroxyl radical formation in a transient cerebral ischemia. Brain Res 762: 240-242, 1997.

4. Abe K, Yuki S, Kogure K. Strong attenuation of ischemic and postischemic brain edema in rats by a novel free radical scavenger. Stroke 19: 480-485, 1988.

5. Nishi H, Watanabe T, Sakurai H, Yuki S, Ishibashi A. Effect of MCI-186 on brain edema in rats. Stroke 20: 1236-1240, 1989.

6. Watanabe T, Egawa M. Effects of an antistroke agent MCl-186 on cerebral arachidonate cascade. J Pharmacol Exp Ther 271: 16241629, 1994.

7. Yamamoto Y, Kuwahara T, Watanabe K, Watanabe K. Antioxidant activity of 3-methyl-1-phenyl-2-pyrazolin-5-one. Redox Rep 2: 333-338, 1996.

8. The Edaravone Acute Brain Infarction Study Group (Chair: Eiichi Otomo). Effect of a novel free radical scavenger, edaravone (MCI-
186), on acute brain infarction. Randamized, placebo-controlled, double-blind study at multicenters. Cerebrovasc Dis 15: 222-229, 2003.

9. Heinel LA, Rubin S, Rosenwasser RH, Vasthare US, Tuma RF. Leukocyte involvement in cerebral infarct generation after ischemia and reperfusion. Brain Res Bull 34: 137-141, 1994.

10. Matsuo Y, Onodera H, Shiga Y. Correlation between myeloperoxidase-quantified neutrophil accumulation and ischemic brain injury in the rat. Effect of neutrophil depletion. Stroke 25: 1469-1475, 1994.

11. Connolly ES, Winfree CJ, Springer TA, Naka Y, Pinsky DJ. Cerebral protection in homozygous null ICAM-1 mice after middle cerebral artery occlusion. J Clin Invest 97: 209-216, 1996.

12. Rothlein R, Czajkowski M, O’Neill MM, Marlin SD, Mainlofi E, Merluzzi VJ. Induction of intracellular adhesion molecule 1 on primary and continuous cell lines by proinflammatory cytokines. J Immunol 141: 1665-1669, 1988.

13. Mori $T$, Asano $T$, Matsui $T$, et al. Intraluminal increase of superoxide anion following transient focal cerebral ischemia in rats. Brain Res 816: 350-357, 1999.

14. Lipton P. Ischemic cell death in brain neurons. Physiol Rev 79: 1431-1568, 1999.

15. Walder CE, Green SP, Darbonne WC. Ischemic stroke injury is reduced in mice lacking a functional NADPH oxidase. Stroke 28: 2252-2258, 1997.

(C) 2006 The Japanese Society of Internal Medicine http://www.naika.or.jp/imindex.html 